medical decision-making in case of disproportions between the large number of victims and the restricted capacities of the medical service. The ISS is a multimedia, hypertext, computer program which is intended to retrieve the necessary information to provide accurate timely diagnosis and to choose optimal treatment for burn patients, especially in mass flame disasters.

Using this system, a physician can retrieve data needed for determination of burn shock severity and shock prognosis on the basis of clinical, functional, and laboratory data as well as the details of optimal anti-shock therapy. The ISS also allows retrieval of required information on the methods of respiratory function, restoration in thermo-chemical injuries of the respiratory system, methods of parenteral and enteral probe feeding, modern tactics of skin plasty, necessary information for triage, and medical aid to victims at the scene, as well as at medical evacuation staging, particularly for specialized hospitals. It includes hundreds of color videoimages of victims with burns of different severities and localization, a glossary of more than 700 terms, and audio support and digital videoclips that illustrate different treatment methods in acute and subsequent stages of burn illness.

The search for necessary information can be accomplished by means of "keywords." In-context-stipulated search with logical conditions also is available. The system can be used not only as an electronic guide for selection of medical actions in cases of disaster, but also can be implemented as a very good visual aid for training. More detailed information on the ISS application can be obtained from the Central Institute of Traumatology and Orthopaedics (Facsimile: 095-154-3139).

Key Words: burns; decision; disaster; information support; multimedia training

\section{An Introduction to the Global Health Disaster Network (GHDNet)}

G. Ocbi; Y. Sbirakawa; K. Nitta; Y. Watob; E. Pretto; $A$. Sekikawa; R.E. La Porte

Department of Emergency Medicine, Ehime University School of Medicine, Ehime, Japan and the Safar Center for Resuscitation Research, University of Pittsburgh, Pittsburgh, Pennsylvania USA

In May 1995, the Global Health Disaster Network (GHDNet) was started with the concept of a disasteroriented project of the Global Health Network (GHNet) at the University of Pittsburgh. The goal of the GHDNet is networking people who are involved in disaster management. Those with various backgrounds, i.e., health-care professionals in disaster and emergency medicine, paramedics and firefighters, public health specialists, are welcome to the network. Being started primarily in Japan, the project will be extended to the United States and the rest of the world. The GHDNet project in Japan consists of following three components: 1) Establishment of $W W W$ bome pages-In July 1995, the GHDNet Home Page (http://hypnos.m.ehime-u. ac.jp/GHDNet) was launched as the first Japanese home page focused on disaster and emergency medicine.
Moreover, we have helped to start more than 20 home pages for disaster related organizations and individuals. The WADEM home page (http://hypnos.m.ehime-u. ac.jp/GHDNet/WADEM) is one of the home pages on our server;

2) Networking people by mailing lists-We started four mailing lists: The Mailing List for Disaster and Emergency Medicine; The Mailing List for Fire and Disaster Prevention; The Mailing List for Japan Red Cross; and The Disaster and Public Health Mailing List. We also are active members of the World NGO Network (WNN) and Inter C-Net which are mailing lists for NGO activities and for public officers in charge of disaster management; and

3) Networking people through medical associations-We maintain home pages for the following medical associations: The World Association for Disaster and Emergency Medicine (WADEM); The Japanese Association for Acute Medicine; and The Japanese Association for the Surgery of Trauma.

It will be our mission to make a link from WWW home pages for these associations and mailing lists to the classical systems for urgent information transmission, i.e., telephone and facsimile.

Key Words: disaster medicine; Internet; telecommunication; www

\section{Quality Management System Implementation within the Viennese Red Cross Ambulance and Disaster Service}

Christoph Redelsteiner, BSW, EMT-P

Wien, Germany

The Vienna Red Cross (VRC) offers various social and medical services to the Viennese community. To assure quality of care, the VRC has implemented a Quality Management System and accomplished successful ISO 9001 certification in October 1996.

An arm of the VRC is its Ambulance Service that provides ALS and BLS Emergency Medical Services, convalescent services, transport of handicapped children and family practitioner-response services. About 130,000 responses are provided per year. Some 600 VRC providers of different levels (drivers, emergency medical technicians, physicians) and from different labor backgrounds (employed, civil servants and volunteers) team up to serve the public.

To further improve quality in such a diverse organizational structure, special "quality teams" have been established. The team consists of EMS providers, managers, and members from other involved VRC departments, e.g., administration, public relation, billing. The teams also developed a quality assurance system according to the 20 ISO 9001 standards as a basis for further Total Quality Management efforts.

So far, staff members have been trained by internal quality system auditors, internal quality audits have been conducted, and all staff members have received information, education, and training in quality management issues. Specialty trained "Quality Assurance Partners" 